\title{
Diagnostic Value of Serum Biomarkers for Intrahepatic Cholangiocarcinoma
}

\author{
Youdi Li1, Yandi Huang2 and Jiu Chen1 \\ Department of Traditional Chinese Medicinel / Clinical Laboratory², The First Affiliated Hospital, College of Medicine, \\ Zhejiang University, Hangzhou, China
}

\begin{abstract}
Objective: To explore the diagnostic value of carbohydrate antigen 19-9 (CA 19-9) and carcinoembryonic antigen (CEA) for the diagnosis of intrahepatic cholangiocarcinoma (ICC).

Study Design: Case control study.

Place and Duration of Study: First Affiliated Hospital, College of Medicine, Zhejiang University, Hangzhou, China, from June 2016 to March 2018.

Methodology: Patients with ICC $(n=51)$ diagnosed by histological examination after surgery were matched 1:4 to control subjects $(n=204)$ based on age ( \pm 2 years), gender, and ethnicity. Serum CA 19-9 and CEA levels were determined and compared. Receiver operating characteristic (ROC) curves were generated, and areas under the receiver operating characteristic curve (AUC) were calculated. The cutoff values, sensitivities, specificities, and Youden index values were calculated and compared.

Results: The levels of CA 19-9 and CEA were elevated in the ICC patients relative to control subjects ( $p<0.001)$. CA 19-9 and CEA showed diagnostic efficacy for ICC, with AUC values of 0.6916 and 0.6376 , respectively. The cutoff value, sensitivity, specificity, and Youden index for CA $19-9$ were $26 \mathrm{U} / \mathrm{ml}, 58.82 \%, 83.82 \%$, and 0.4262, respectively. The cutoff value, sensitivity, specificity, and Youden index for CEA were $1.95 \mathrm{ng} / \mathrm{ml}, 90.2 \%, 35.29 \%$, and 0.2549 , respectively. The combination of CA 19-9 and CEA exhibited the best diagnostic efficacy, with the sensitivity, specificity, and Youden index being $90.2 \%, 88.24 \%$, and 0.7844 , respectively.

Conclusion: Combined CA 19-9 and CEA levels have diagnostic value for ICC. The combination of CA 19-9 and CEA can assist surveillance and diagnosis of ICC prior to surgery.
\end{abstract}

Key Words: Serum biomarkers, Intrahepatic cholangiocarcinoma, Des-gamma-carboxyprothrombin, Carbohydrate antigen 19-9, Carcinoembryonic antigen, Carbohydrate antigen-125.

How to cite this article: Li Y, Huang Y, Chen J. Diagnostic value of serum biomarkers for intrahepatic cholangiocarcinoma. $J$ Coll Physicians Surg Pak 2019; 29(10):962-6.

\section{INTRODUCTION}

In 2018, liver cancer was predicted, worldwide, to be the sixth most commonly diagnosed cancer as well as the fourth leading cause of cancer. ${ }^{1}$ Intrahepatic cholangiocarcinoma (ICC) is a malignancy located proximal to the second order bile ducts, accounting for $10-20 \%$ of all liver cancers. ${ }^{2}$ The overall incidence of ICC has risen in recent decades and is anticipated to continually increase in the coming decades. ${ }^{3}$ Although relatively rare compared with hepatocellular carcinoma (HCC), ICC is a more aggressive cancer, 4 and initially silent, with patients asymptomatic at early stage. Up to $80 \%$ of patients have advanced stage disease at diagnosis. ${ }^{4}$ Ablation or surgical resection is the only potential curative treatments for early patients, ${ }^{5}$ with liver transplantation, a treatment option for very early patients. ${ }^{6}$ For patients with advanced stage disease, available therapies have

Correspondence to: Jiu Chen, Department of Traditional Chinese Medicine, The First Affiliated Hospital, College of

Medicine, Zhejiang University, Hangzhou, China

E-mail:linyunju@zju.edu.cn

Received: December 21, 2018; Revised: March 24, 2019; Accepted: April 19, 2019 limited effectiveness with median overall survival less than one year. ${ }^{6}$ Surveillance and early diagnosis are extremely important. However, the diagnosis of ICC is challenging even with current imaging techniques. An array of pitfalls to diagnosis can be hidden even in classical images. ${ }^{7}$

Serum is readily accessible and serum biomarkers have proved useful for the diagnosis of multiple cancers. Unfortunately, no specific biomarkers exist for ICC. Desgamma-carboxyprothrombin (DCP) has been shown to have diagnostic efficacy for $\mathrm{HCC}, 8$ but its diagnostic efficacy for ICC has not been established. Carbohydrate antigen 19-9 (CA 19-9), carcinoembryonic antigen (CEA), and carbohydrate antigen-125 (CA-125) have been assessed individually and in combination as biomarkers for cholangiocarcinoma, with the possibility that combined measurement would increase diagnostic efficacy. ${ }^{9}$

Herein, the aim of the study was to explore the diagnostic efficacy for ICC of DCP, CA 19-9, CEA, and CA-125 alone and in combination. The rationale was to identify reliable serum biomarkers for ICC that improved diagnostic efficacy. 


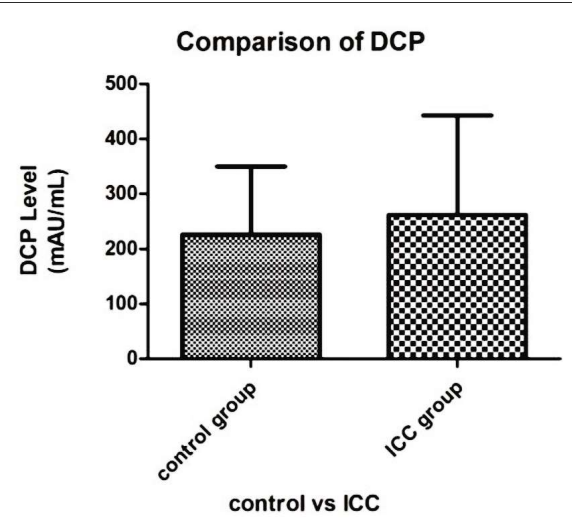

Comparison of CEA

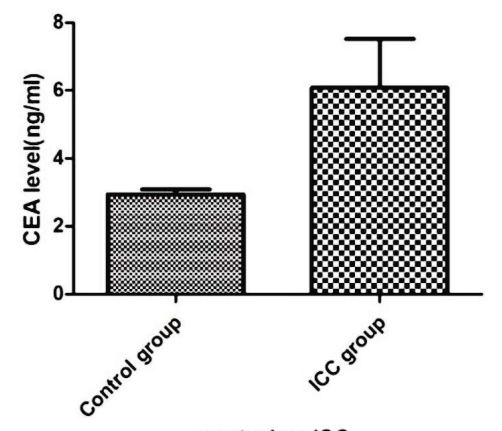

control vs ICC

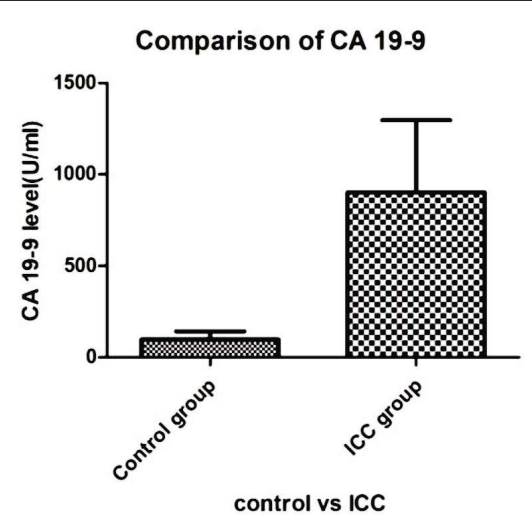

Comparison of CA-125

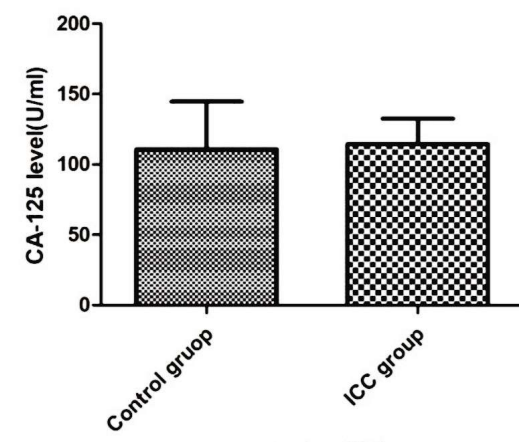

control vs ICC

Figure 1: Comparison of serum levels of DCP, CA 19-9, CEA, and CA-125 in patients with ICC and control subjects.

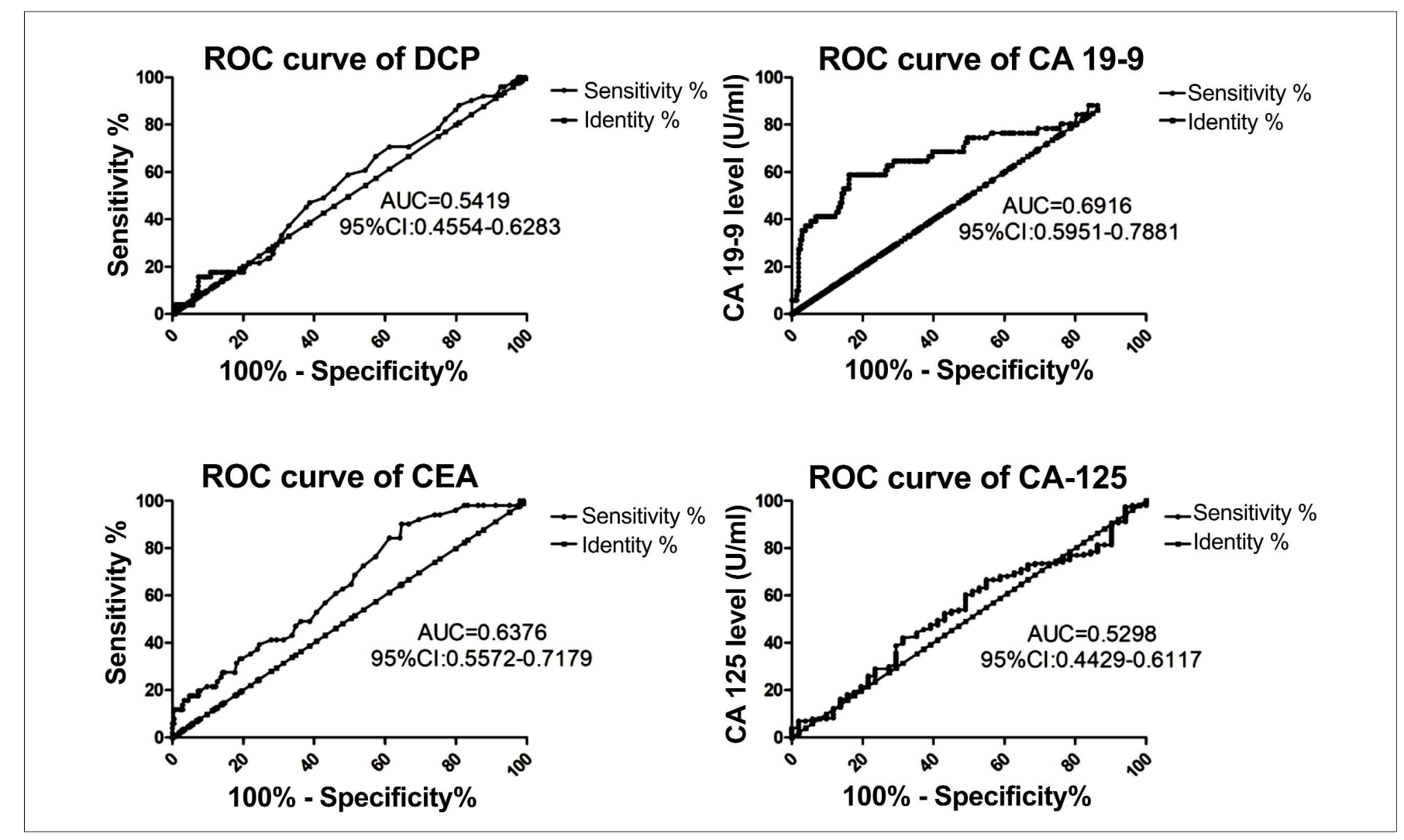

Figure 2: ROC curves for DCP, CA 19-9, CEA, and CA-125 in patients with ICC and control subjects. 


\section{METHODOLOGY}

Patients with ICC diagnosed by histological examination ( $n=51$ ) from June 2016 to March 2018 at First Affiliated Hospital, College of Medicine, Zhejiang University, Hangzhou, China, were matched 1:4 with control subjects $(n=204)$, based on age $( \pm 2$ years), gender (male or female), and ethnicity (xanthoderm). This study was approved by the Ethics Committees of the Hospital. The inclusion criteria were all serum biomarker data (DCP, CA 19-9, CEA, and CA-125) obtained from ICC patients prior to treatment, age being $>18$ years. The exclusion criteria were as follows: previous liver transplantation; vitamin $\mathrm{K}$ or warfarin within two weeks of blood sample collection; malignancies other than ICC; unconfirmed nodules in the liver when the patient refused surgery; or prior treatment. For the control, all the data were collected from healthy participants.

All serum samples were collected and tested in the same laboratory. Serum samples were stored at $-80^{\circ} \mathrm{C}$ until used for testing. Serum levels of CA 19-9,CEA, and CA-125 were analysed within 4 hours after collection, using an Abbott i2000SR automatic analyser and associated kits (Abbott Diagnostics, Chicago, Illinois, U.S.A.), according to the manufacturer's instructions. Serum levels of DCP were measured by chemiluminescent enzyme immunoassay (Lumipulse ${ }^{\circledR} \quad G$ PIVKA- II; FUJIREBIO INC., Japan), according to the manufacturer's instructions. The author had collected and analysed the data. All statistical analyses were conducted using GraphPad Prism 5 (GraphPad Software Inc., San Diego, CA, US). $P<0.05$ was considered significant. Categorical data were expressed as percentage values. Receiver operating characteristic (ROC) curve analysis was used to assess the diagnostic efficacy of ICC. Areas under the receiver operating characteristic curve (AUC) were calculated based on the ROC curves.

\section{RESULTS}

Serum biomarker levels were measured and compared between the control and ICC groups. As shown in Figure 1 , the levels of CA 19-9 and CEA were elevated in the ICC group compared with controls $(p<0.001$ and 0.002 , respectively). There were no significant differences in the levels of DCP or CA-125 between the control and ICC groups ( $p=0.355$ and 0.510 , respectively). ROC curves plotted based on serum biomarker, CA 19-9 and CEA levels (Figure 2) showed diagnostic efficacy for ICC, with AUC values greater than 0.5 (0.691 and 0.637

Table I: The cutoff value, sensitivity, specificity and Youden index value for CA 19-9 and CEA, alone and in combination.

\begin{tabular}{l|c|c|c|c}
\hline Tumor biomarker & Cutoff value & $\begin{array}{c}\text { Sensitivity } \\
(\%)\end{array}$ & $\begin{array}{c}\text { Specificity } \\
(\%)\end{array}$ & $\begin{array}{c}\text { Youden } \\
\text { index }\end{array}$ \\
\hline CA 19-9 & $26 \mathrm{U} / \mathrm{ml}$ & 58.82 & 83.82 & 0.4262 \\
CEA & $1.95 \mathrm{ng} / \mathrm{ml}$ & 90.2 & 35.29 & 0.2549 \\
CA 19-9 and CEA & & 90.2 & 88.24 & 0.7844 \\
\hline \multicolumn{4}{l}{ For CA 19-9 and CEA, the levels exceed both of the cutoff values. }
\end{tabular}

respectively). DCP and CA-125 demonstrated no diagnostic efficacy, with AUC values close to 0.5 (0.541 and 0.529 , respectively). The cutoff values, sensitivities, specificities, and Youden index values are shown in Table I. Compared with individual biomarkers, the combination of CA 19-9 and CEA increased the diagnostic efficacy. The combination of CA 19-9 and CEA exhibited excellent diagnostic efficacy, with a Youden index of 0.784 .

\section{DISCUSSION}

ICC is an aggressive malignancy with an increasing incidence for which clinical diagnostic biomarkers do not exist. 1,2,4,7,10 Most of the previous studies explored the diagnostic biomarkers for cholangiocarcinoma other than ICC alone. Li et al. found that the microRNA-laden extracellular vesicles could be used for diagnosis of cholangiocarcinoma with the sensitivity of $67 \%$ and specificity of $96 \%$, but it is far from clinical application. ${ }^{11}$ This study was designed to explore the diagnostic efficacy of serum biomarkers for ICC common to clinic usage. With ease of implementation, good reproducibility, objectivity, and non-invasiveness, CA 19-9 and CEA in combination showed the sensitivity of $90.2 \%$ and specificity of $88.24 \%$. The AUC and Youden index are the most frequently used parameters for measurement of diagnostic efficacy, 12,13 with higher values indicating superior efficacy. 14 In this study, both methods were used to assess the diagnostic efficacy of serum biomarkers in patients with ICC.

DCP has been shown to have excellent diagnostic efficacy for HCC, 8 while CA-125 is a well-known tumor marker for ovarian cancer. 15 No previous study has assessed the diagnostic efficacy of DCP for ICC; and herein, we demonstrate that DCP is not a useful biomarker for ICC. If liver nodules are found by imaging and DCP levels are normal, other pathologies like ICC should be considered because HCC and ICC consist of almost all primary liver cancers. ${ }^{3}$ Cholangiocarcinoma is divided into ICC and extrahepatic cholangiocarcinoma (ECC) based on anatomical position. CA-125 has been shown to have diagnostic efficacy for cholangiocarcinomas other than ICC. In this study, neither DCP nor CA-125 had diagnostic efficacy for ICC. There were no significant differences in the levels of DCP or CA-125 between the control and ICC groups with AUC values for both close to 0.5 . The difference between the results reported herein and those previously reported for CA-125 may be the difference in anatomical location. It is worth noting that the five-year survival rate is much poorer for ICC than ECC with the incidence of ICC increasing; while the incidence of ECC decreasing in western countries. ${ }^{16}$ The characteristics of cholangiocarcinomas differ, based on anatomical position with CA-125 a better likely marker for ECCs. 
CA $19-9$ is widely used for clinical assessment of pancreatic cancer while CEA has been used for the diagnosis of colon, pancreatic, and gastric cancer. 17 In this study, both CA 19-9 and CEA were shown to have diagnostic efficacy for ICC. Both of the AUC values were greater than 0.5. For CA 19-9, the cutoff value, sensitivity, specificity, and Youden index were $26 \mathrm{U} / \mathrm{ml}$, $58.82 \%, 83.82 \%$, and 0.4262 , respectively. For CEA, the cutoff value, sensitivity, specificity, and Youden index were $1.95 \mathrm{ng} / \mathrm{ml}, 90.2 \%, 35.29 \%$, and 0.254 , respectively.

The normal reference range of CA 19-9 and CEA were 0-37 U/ml and $0-5 \mathrm{ng} / \mathrm{ml}$, respectively, according to the manufacturer's instructions. As both of CA 19-9 and CEA are non-specific serum biomarkers for digestive system neoplasms, it is necessary to set the exact cutoff valve for one kind of specific tumor. In this study, the cutoff values of CA 19-9 and CEA for ICC were $26 \mathrm{U} / \mathrm{ml}$ and $1.95 \mathrm{ng} / \mathrm{ml}$, respectively. Compared with the reference range of the manufacturer's instructions, the cutoff values were much lower than the upper limit of the reference range, which means that for ICC, the cutoff values should be lower than other digestive system neoplasms such as pancreatic, colon, pancreatic, and gastric cancer.

For ICC, CA 19-9 has excellent diagnostic efficacy in specificity and CEA has excellent diagnostic efficacy in sensitivity. The CA 19-9 had better specificity than CEA $(83.82 \%$ vs. $35.29 \%)$; while CEA had better sensitivity than CA $19-9$ (90.2\% vs. 58.82\%). The Youden index, (defined as the maximum value of sensitivity+specificity-1), fits well into the ROC framework. ${ }^{18}$ The Youden index values for CA 19-9 and CEA were 0.426 and 0.254 , respectively, which are not very high. The combination of high sensitivity for CEA and high specificity for CA 19-9 markedly improved diagnostic efficacy. The sensitivity, specificity, and Youden index of the combination was $90.2 \%, 88.24 \%$, and 0.7844 , respectively.

Although patients with ICC were matched to controls based on age, gender, and ethnicity, the selection bias may have existed as ICC is relatively rare. Future prospective analysis of larger numbers of patients is necessary to confirm the results of this investigation.

\section{CONCLUSION}

With high diagnostic efficacy, ease of implementation, good reproducibility, objectivity, and non-invasiveness, CA 19-9 and CEA in combination should be considered readily available adjuncts to imaging for surveillance and the diagnosis of ICC before surgery. If liver nodules are found by imaging in the liver and both of CA 19-9 and CEA levels are abnormal while DCP level is normal, ICC is mostly likely to be diagnosed before surgery and further treatment could be performed based on this diagnosis.

\section{ETHICAL APPROVAL:}

The study was approved prior to initiation of the research work by the Ethics Committees of the First Affiliated Hospital, College of Medicine, Zhejiang University.

\section{PATIENTS' CONSENT:}

As a retrospective cohort study, a waiver for informed consent was obtained and all the data from patients were analysed anonymously.

\section{CONFLICT OF INTEREST:}

Authors declared no conflict of interest.

\section{AUTHORS' CONTRIBUTION:}

YL: Responsible for revising it critically for important intellectual content.

$\mathrm{YH}$ : Responsible for the analysis of data for the work. JC: Responsible for the conception and design of the work; and for the final approval of the version to be published.

\section{REFERENCES}

1. Bray F, Ferlay J, Soerjomataram I, Siegel RL, Torre LA, Jemal A. Global cancer statistics 2018: Globocan estimates of incidence and mortality worldwide for 36 cancers in 185 countries. CA Cancer J Clin 2018; 68:394-424.

2. Gupta A, Dixon E. Epidemiology and risk factors: Intrahepatic cholangiocarcinoma. Hepatobiliary Surg Nutr 2017; 6:101-4.

3. Massarweh NN, El-Serag HB. Epidemiology of hepatocellular carcinoma and intrahepatic cholangiocarcinoma. Cancer Control 2017; 24:1073274817729245.

4. Chun YS, Javle M. Systemic and adjuvant therapies for intra-hepatic cholangiocarcinoma. Cancer Control 2017; 24: 1073274817729241.

5. Currie BM, Soulen MC. Decision making: Intra-arterial therapies for cholangiocarcinoma-TACE and TARE. Semin Intervent Radiol 2017; 34:92-100.

6. Rizvi S, Khan SA, Hallemeier CL, Kelley RK, Gores GJ. Cholangiocarcinoma-evolving concepts and therapeutic strategies. Nat Rev Clin Oncol 2018; 15:95-111.

7. Chung YE, Kim MJ, Park YN, Choi JY, Pyo JY, Kim YC, et al. Varying appearances of cholangiocarcinoma: Radiologicpathologic correlation. Radiographics 2009; 29:683-700.

8. Chen J, Wu G, Li Y. Evaluation of serum des-gamma-carboxy prothrombin for the diagnosis of hepatitis $B$ virus-related hepatocellular carcinoma: A meta-analysis. Dis Markers 2018; 2018:8906023.

9. Malaguarnera G, Paladina I, Giordano M, Malaguarnera M, Bertino G, Berretta M. Serum markers of intrahepatic cholangiocarcinoma. Dis Markers 2013; 34:219-28.

10. Magistri P, Tarantino G, Serra V, Guidetti C, Ballarin R, Di Benedetto F. Liver transplantation and combined hepatocellular-cholangiocarcinoma: Feasibility and outcomes. Dig Liver Dis 2017; 49:467-70.

11. Sirica AE, Gores GJ, Groopman JD, Selaru FM, Strazzabosco M, Wang XW, et al. Intrahepatic cholangiocarcinoma: Continuing challenges and translational advances. Hepatology 2019; 69: 1803-15.

12. Alemayehu D, Zou KH. Applications of ROC analysis in medical research: Recent developments and future directions. Acad Radiol 2012; 19:1457-64.

13. Chen F, Xue Y, Tan MT, Chen P. Efficient statistical tests to compare Youden index: Accounting for contingency correlation. Stat Med 2015; 34:1560-76. 
14. Xiong X, Yan P, Gao C, Sun Q, Xu F. The value of contrastenhanced ultrasound in the diagnosis of cesarean scar pregnancy. Biomed Res Int 2016; 2016:4762785.

15. Bottoni P, Scatena R. The role of CA 125 as tumor marker: Biochemical and clinical aspects. Adv Exp Med Biol 2015; 867:229-44.

16. Kayhanian $\mathrm{H}$, Smyth $\mathrm{EC}$, Braconi $\mathrm{C}$. Emerging molecular targets and therapy for cholangiocarcinoma. World J Gastrointest Oncol 2017; 9:268-80.

17. Zhang Y, Yang J, Li H, Wu Y, Zhang H, Chen W. Tumor markers CA19-9, CA242 and CEA in the diagnosis of pancreatic cancer: A meta-analysis. Int J Clin Exp Med 2015; 8:11683-91.

18. Yin J, Tian L. Joint confidence region estimation for area under ROC curve and Youden index. Stat Med 2014; 33:985-1000. .......... 